\title{
Prioritized Call Management Scheme with Adjustable Guard Channels
}

\author{
Ezenugu Isaac A. ${ }^{1}$, Eke James ${ }^{2}$, Alor Michael O. ${ }^{2}$ \\ ${ }^{1}$ Department of Electrical/Electronic Engineering, Imo State University, Owerri, Nigeria \\ ${ }^{2}$ Department of Electrical/Electronic Engineering, Enugu State University of Science and Technology Enugu, Nigeria
}

Email address:

isaac.ezenugu@yahoo.com (Ezenugu I. A.)

\section{To cite this article:}

Ezenugu Isaac A., Eke James, Alor Michael O. Prioritized Call Management Scheme with Adjustable Guard Channels. International Journal of Theoretical and Applied Mathematics. Vol. 3, No. 3, 2017, pp. 100-105. doi: 10.11648/j.ijtam.20170303.11

Received: October 25, 2016; Accepted: December 27, 2016; Published: April 25, 2017

\begin{abstract}
Network congestion and signal quality degradation are the major problems of the Global System for Mobile communication (GSM), most especially as the number of customers increases. Consequently, there is a pressing demand for further research to improve network performance. However, congestion in various systems has always been tackled with various attempts, all of which falls in either the congestion avoidance category or congestion management category. Congestion avoidance has however been adjudged the best scheme for controlling network congestion and this is the approach employed in this research work. This research work is focused on determination of a busy BTS(s) within different GSM network areas, and how to manage the BTS(s) in order to reduce call blocking/dropping to the barest minimum. Traffic intensity value varies from one BTS to another, as it is directly proportional to the number of GSM users that make or receive calls within a particular period of time. This paper proposes a new scheme that will consider the number of reserved channels within a particular BTS. The scheme also considers the following; available channels, offered traffic, new call arrival rate, handoff call arrival rate and mean call duration. The continuous-time single dimensional birth-death process is also adopted to model and analyze the performance of this scheme. Moreover, MATLAB was used for simulation of the analytical equations which were obtained from the proposed state transition diagram. However, analytical method and simulation were used in this research work.
\end{abstract}

Keywords: Handoff Failure, Handoff Queue, Mobile Networks, Quality of Service, Call Drop

\section{Introduction}

With GSM communications, a user that initiates or receives a call may move around the area covered by the network. If the mobile user moves from one cell to another, and the call from/to the user has not finished, the network has to handoff the call from one cell to another at the cell boundary crossing without user's awareness of handoff and without much degradation of the service quality. As the demand for wireless communication systems by the users keep increasing, a good quality of service (QoS) is required to manage the incoming new calls and handoff calls more efficiently. Radio resource management (RRM) plays a vital role in cellular networks to efficiently utilize the limited radio resources while guaranteeing the required QoS for mobile users. In the GSM system, the Mobile Stations (MS) communicates with the Base Station System (BSS) via the RF air interface. The Base Station System (BSS) consists of a Base Transceiver Station
(BTS), and a Base Station Controller (BSC). In most cases, several BTS are located at the same site, this gives rise to two (2) to four (4) sectored cells around a shared antenna tower. BSCs are often connected to BTS via microwave links. The BSC to BTS link is called the Abis interface. Usually about 20 to 30 BTS are controlled by a single BSC. Several BSS will be reporting to the same Mobile Switching Center (MSC) which controls the traffic among many different cells. Each (MSC) is assigned a Visitors Location Register (VLR) where mobile stations which are outside their home cell are listed, so as to enable the network to be aware where to find them. The MSC will also be connected to the Home Location Register (HLR), the Authentication Center (AUC), and the Equipment Identity Register (EIR). These connections of the MSC are to ensure that the system can verify that the users and equipment are legal subscribers. This helps avoid the use of stolen or fraud mobiles. Within the system there are also facilities for Operations and Maintenance (OMC) as well as Network Management (NMC) organizations. The MSC also has the 
interface to other networks such as Private Land Mobile Networks (PLMN) and Public Switched Telecommunication Network (PSTN) and Integrated Service Digital Network (ISDN) [1].

Several attempts had been made to forestall and manage the congestion in mobile networks like the GSM network which includes channel borrowing, cell-splitting, cell sectoring, development of micro-cells, dynamic channel allocation and deployment of soft handover schemes [2-4]. The techniques of controlling congestion employed so far in all systems have always in a general view, being focused on two principles. These principles are either to reject excessive traffic to prevent overload from occurring or diverting excess load if overload occurs. All the proposed techniques in the literatures had therefore been centered on these two principles. Attempt is made to use a combined scheme consisting of load balancing technique with a known efficient CAC scheme in order to reduce the rate of network congestion and signal quality degradation. Hence, the call performance has to be evaluated.

Call admission control (CAC) is a fundamental mechanism used for QoS provisioning in a network [5-7]. It is the rule to admit requested calls maintaining the QoS for the system. $\mathrm{CAC}$ is a key element in the provision of guaranteed QoS in wireless networks. Admission control decision is made using a traffic descriptor that specifies traffic characteristics and QoS requirements. A new call request is accepted if there is free channel in the network resource, and also if the call meets the QoS requirements of new calls without disrupting the QoS for the already supported calls. Too many calls lead to a situation where the mutual interference between the connections degrades the QoS for the new call as well as for the ongoing connections. Therefore, admission control plays a very important role in providing the user with the requested QoS as well as making an efficient use of the available capacity and preventing the system from an outage situation due to overloading [8-11]]. An accepted call that has not completed in the current cell may have to be handed off to another BTS.

During the process, the call may not be able to gain a channel in the new BTS to continue its service due to the limited resource in wireless networks, which will lead to call dropping. Relatively, new calls and handoff calls can be treated differently in terms of resource allocation. Since dropping a call in progress is more annoying than blocking a new call request, handoff calls are typically given higher priority than new calls in access to the wireless resources. This preferential treatment of handoffs increases the blocking of new calls and hence degrades the bandwidth utilisation [12]. Considering the users' freedom of movement, mobility management becomes a major issue of interest. For efficient call connection service to a customer, the location of a mobile user must be identified within a certain time. The network has to keep tracing the mobile users and allocate enough resource to provide continuing service as the mobile is in motion. This is done without user's awareness of the changes in the network facility. There are two operations used to carry out the mobility management analysis on wireless systems. These are: location update and terminal paging. Location update relates to the process where a mobile user informs the network where it is, while terminal paging is a process that the network attempts to locate a mobile user in the area it was last reported. This area is known as uncertainty area. Both processes which invoke signaling traffic in the signaling networks are used by both processes [13-15]. It is stated in [15], that the more frequent the location update, the higher the signaling traffic for location updates, while the smaller the uncertainty area, hence the lower the signaling traffic for terminal paging. In the other hand, the less frequent the location update, the lower the signaling traffic for the location updates, and the larger the uncertainty area, hence the higher the signaling traffic for terminal paging. It is therefore necessary to try to balance these two kinds of signaling traffic for effective mobility management. In order to achieve a good design for mobility management, it is required that the researcher should quantify the total signaling traffic and also carry out effective optimization. Two traffic request types are considered in this analysis. These are the new calls and handoff call requests. Also this is not a multiclass model in that only class 1 (voice) is considered.

\section{Methods and Algorithms}

Only two techniques; Analytical modeling and Simulation, will be adopted for the modeling and testing approaches. The need for the combination of these two techniques arises since either of the techniques cannot be relied upon to give the needed conclusion. A simulation environment in MATLAB will be built and used for the analysis. The results of the simulation will be presented in tables and graphs plots along with report on the performance variations between the analytical/computed data and the simulation results. The empirical modeling approach is not employed to model the propagation parameters since access to the base stations is restricted.

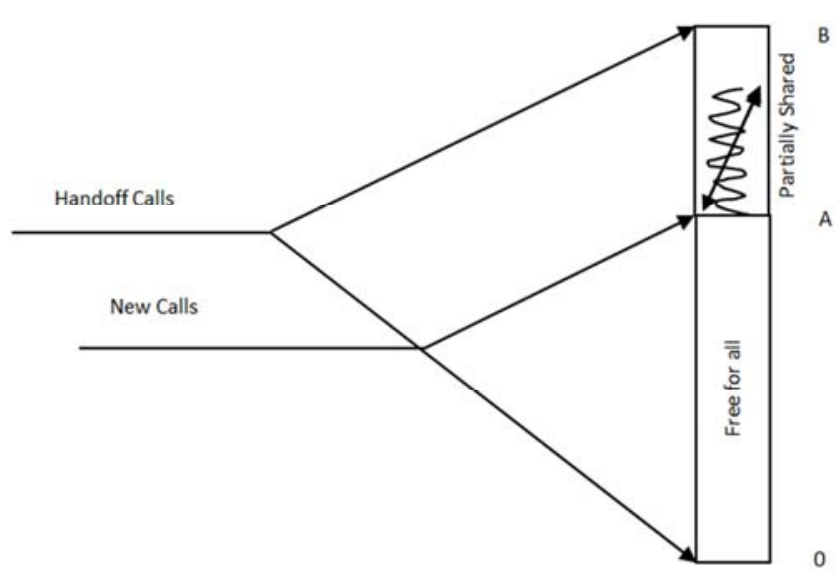

Figure 1. Proposed model.

The model shown in figure 1 is divided into two parts; free for all and shared channels. The "free for all" part does not prioritise any call, be it new call or handoff call. The "partially shared" part accepts both new calls and handoff calls but gives 
priority to handoff calls, but uses a probability factor $(\gamma)$ to determine the percentage of the free channels within this part before priority is given to handoff calls.

Meanwhile, the queuing model for the new proposed scheme is as shown in figure 2. In the queuing model, the new calls can queue between level " 0 " and some part of level "B" with a probability factor $(\gamma)$, while handoff calls can queue between level " 0 " and level "B".

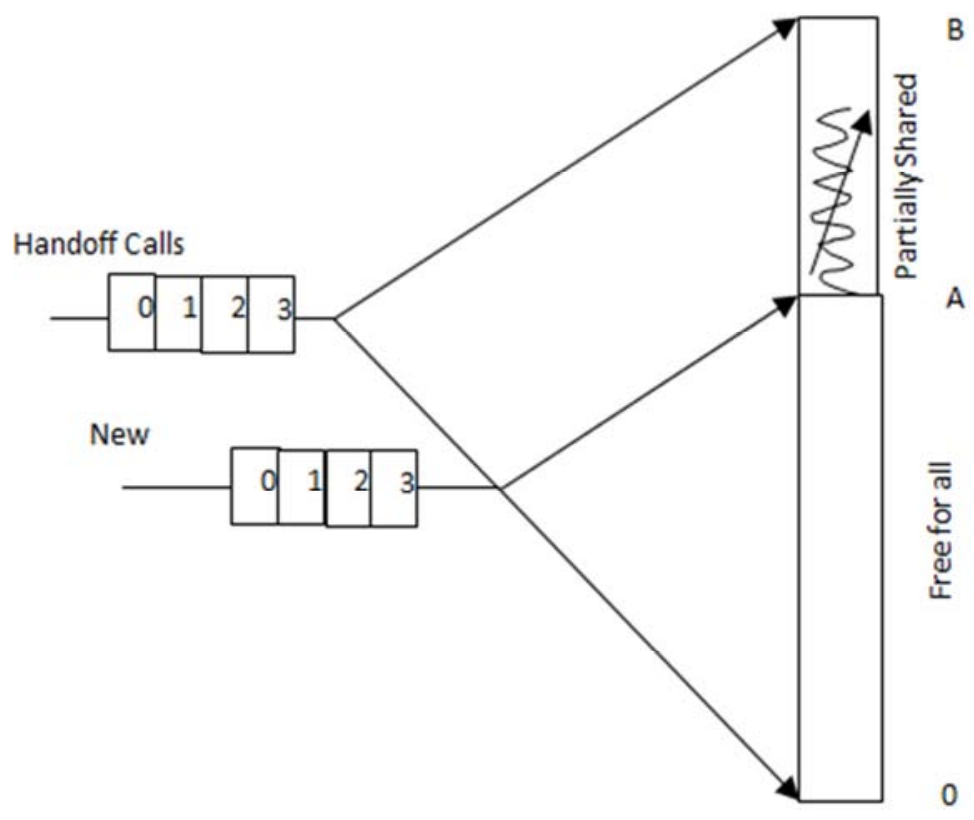

Figure 2. Queuing model of the scheme.

Figure 3 shows the state transition diagram for the newly proposed model shown in figure 1, and its queuing model shown in figure 2. This state transition diagram throws more insight on how different calls are being accepted within a particular range of channels. The symbols in figure 3 and their representations are as follow;

i. $\lambda_{\mathrm{n}}$ stands for new call arrival rate,

ii. $\lambda_{\mathrm{h}}$ stands for handoff arrival rate,

iii. $\gamma$ stands for relative probability factor,

iv. $\mu$ stands for service rate,

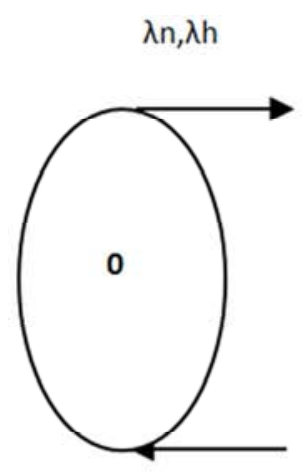

$\mu$

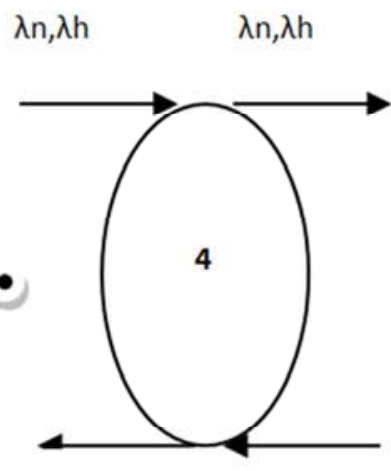

$\mu$

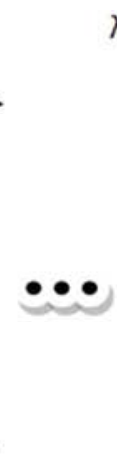

$\mu$

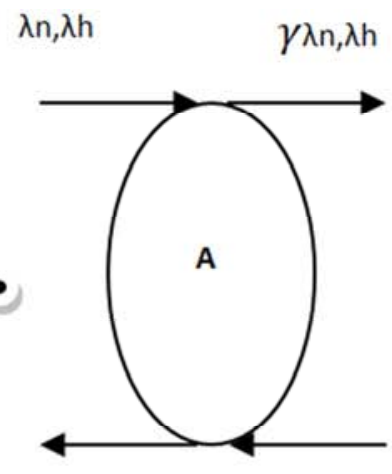

$\mu$

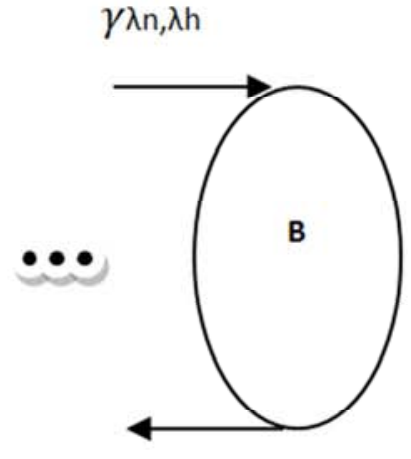

$\mu$

Figure 3. State transition diagram of the scheme.

Meanwhile, the different equations for the two parts of the model are;

$$
\begin{gathered}
\mathrm{i} \mu \mathrm{P}(\mathrm{i})=\frac{\left(\lambda_{\mathrm{N}}+\lambda_{\mathrm{H}}\right)^{\mathrm{i}}}{\mu^{\mathrm{i}} \mathrm{i} !} \mathrm{P}_{0} \leq \mathrm{i} \leq \mathrm{A} \\
\mathrm{i} \mu \mathrm{P}(\mathrm{i})=\frac{\left(\lambda_{\mathrm{N}}+\lambda_{\mathrm{H}}\right)^{\mathrm{i}}}{\mu^{\mathrm{i}} \mathrm{i} !} \times \frac{\left(\gamma \lambda_{\mathrm{N}}+\lambda_{\mathrm{H}}\right)^{\mathrm{i}-\mathrm{A}}}{\mu^{(\mathrm{i}-\mathrm{A})}(\mathrm{i}-\mathrm{A}) !} \mathrm{P}_{0} \quad \mathrm{~A} \leq \mathrm{i} \leq \mathrm{B}
\end{gathered}
$$

Therefore, the normalisation condition is given as

$$
\sum_{i=0}^{B} \mathrm{P}(i)=1
$$

Using equations 1 and 2 recursively along with the normalisation condition, the steady-state probability $P(i)$ is easily found as follows:

$$
P_{i}=\left\{\begin{array}{c}
\frac{\left(\lambda_{n}+\lambda_{h}\right)^{i}}{\mu^{i} i !} P_{0} 0 \leq i \leq A \\
\frac{\left(\lambda_{n}+\lambda_{h}\right)^{i}}{\mu^{i} i !}+\frac{\left(\gamma \lambda_{n}+\lambda_{h}\right)^{i-A}}{\mu^{(i-A)}(i-A) !} P_{0} A \leq i \leq B
\end{array}\right.
$$

Where the probability at state zero or initial probability $\left(\mathrm{P}_{0}\right)$ is given as follows: 


$$
P_{0}=\left[\sum_{i=0}^{A} \frac{\left(\lambda_{n}+\lambda_{h}\right)^{i}}{\mu^{i} i !}+\sum_{i=(A+1)}^{B} \frac{\left(\lambda_{n}+\lambda_{h}\right)^{i}}{\mu^{i} i !} \times \frac{\left(\gamma \lambda_{n}+\lambda_{h}\right)^{i-A}}{\mu^{(i-A)}(i-A) !}\right]^{-1}
$$

Meanwhile, figure 4 represents the flow-chart of the proposed call management scheme. It contains the sequential activities of the new scheme.

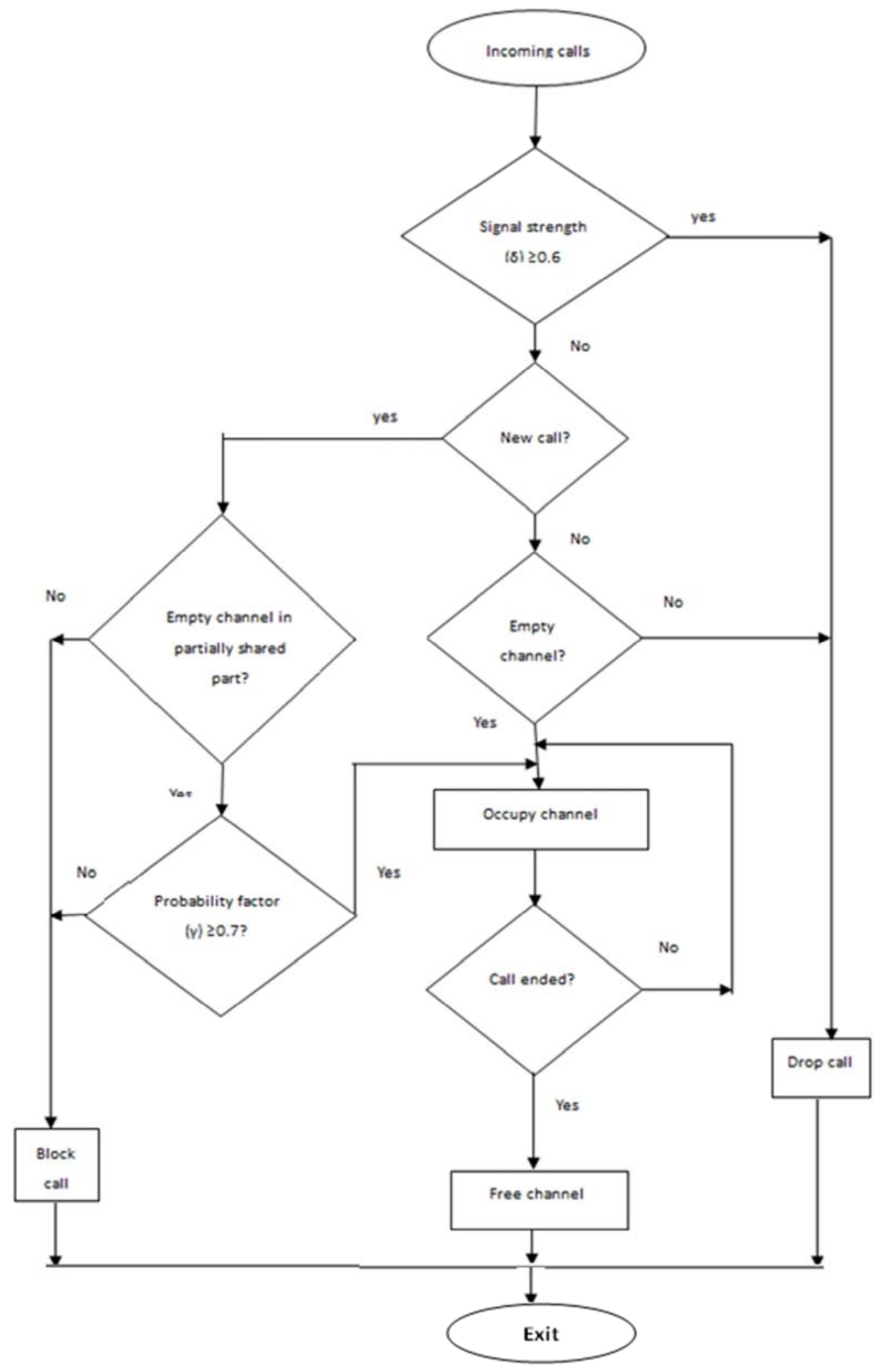

Figure 4. Flow chart of the proposed scheme. 


\section{Results and Discussion}

The different parameters and their values or range of values are as presented in Table 1.

Table 1. Proposed system parameters and their value(s) for simulation.

\begin{tabular}{ll}
\hline PARAMETERS & VALUES \\
\hline No. of channels $(\mathrm{C})$ & $5-34$ \\
New call arrival rate $\left(\lambda_{\mathrm{n}}\right)$ & $1.5(/ \mathrm{s})$ \\
Handoff arrival rate $\left(\lambda_{\mathrm{h}}\right)$ & $2(/ \mathrm{s})$ \\
Signal strength factor $(\delta)$ & $0.6-0.9$ \\
Reserved $($ Guard) channels $(\mathrm{R})$ & Varied from $2-8$ \\
Mobility factor $(\alpha)$ & 0.9 \\
New call duration $\left(\right.$ mean $\left.1 / \mu_{\mathrm{n}}\right)$ & $1-3(\mathrm{~s})$ \\
Handoff call duration $\left(\right.$ mean $\left.1 / \mu_{\mathrm{h}}\right)$ & $2-5(\mathrm{~s})$ \\
New/Handoff traffic ratio & Varied from $2-5$ \\
\hline
\end{tabular}

The following graphs presented in Figures 5 and 6 were obtained through MATLAB simulation using all the parameters' values that were shown in Table 1.

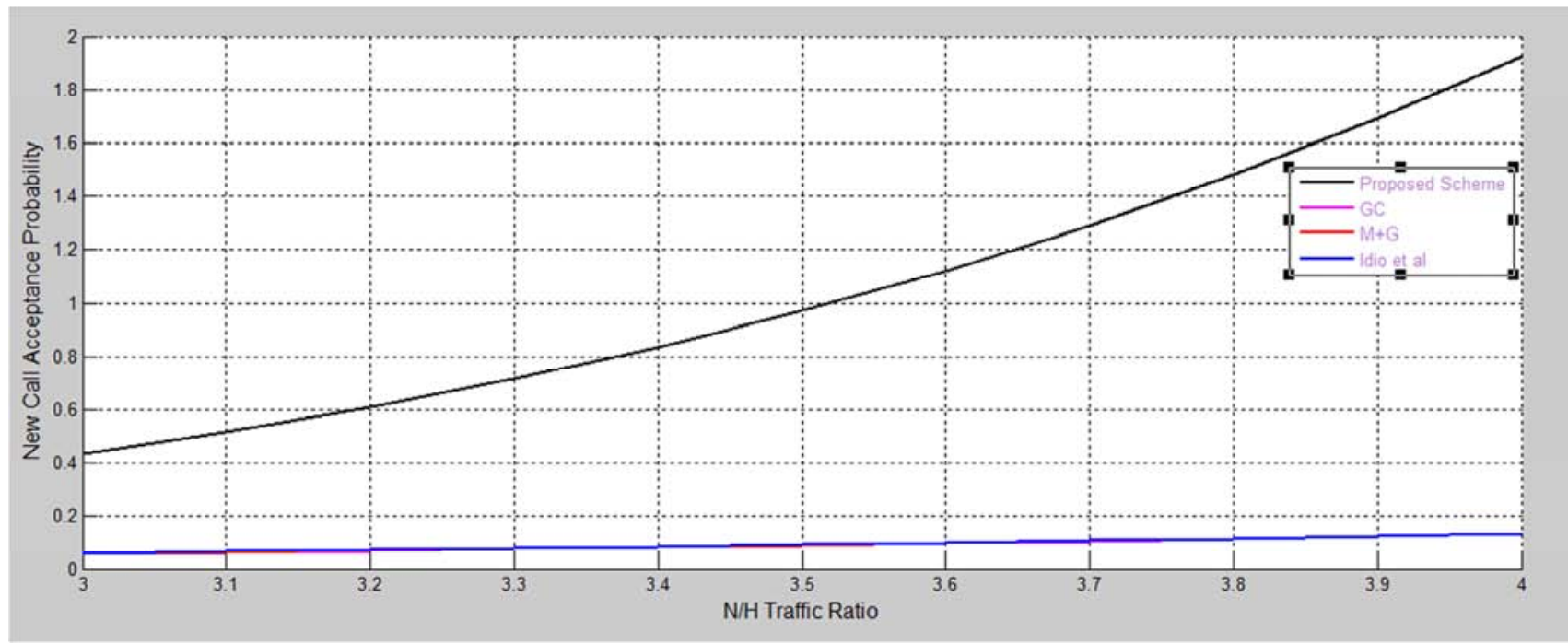

Figure 5. Effect of new/handoff ratio on new call acceptance probability for simulation I.

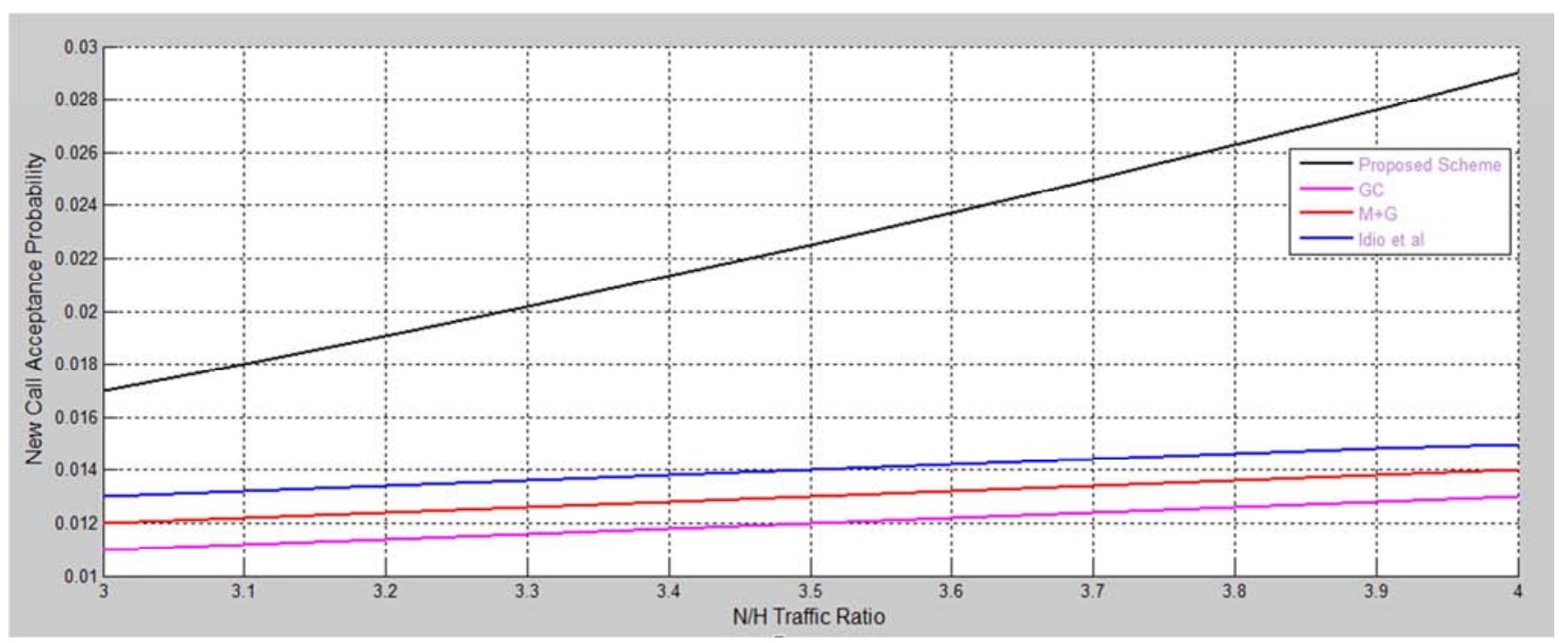

Figure 6. Effect of new/handoff ratio on new call acceptance probabilities for simulation II.

\section{Conclusion}

Having studied different schemes for managing new calls and handoff calls especially those of non-prioritised and prioritised schemes, therefore, the researcher is of the opinion that the available channels need better management scheme. Also, the 
analysis on the performance of different handoff and new calls management schemes in mobile networks has been done in this work. Hence a new scheme was proposed. The proposed scheme is a prioritised one which ensures better channel utilization at all time. The performance of this new scheme in terms of call blocking probability was carried out using MATLAB software. The different results obtained through simulation of both the existing and proposed new/handoff management schemes were presented and compared. With reference to figures 5 and 6 , the performance of the new scheme proved to be better when compared with other existing schemes. The proposed scheme is based on the idea that reserved or guard channels could be idle while new calls are being rejected. A relational probability factor $(\gamma)$ is always used to check if new calls can be allocated channels from the partially reserved channels. Hence, this proposed scheme serves as a hybrid scheme which considers both new calls and handoff calls.

\section{References}

[1] Donaldson A. E., Constance K., and Ikenna C. D. (2016): Cluster-Based Call Acceptance Principle for Optimum Reduction of Call Failures in a GSM Network System; Mathematical and Software Engineering, Vol. 2, No. 2, 48-56. Varspsilon Ltd, http://varepsilon.com.

[2] Rappaport, T. S. (1996). Wireless Communications: Principles and Practice. Upper Saddle River, N. J. London, Prentice Hall PTR, Prentice Hall International.

[3] Ahmed, J. U., Hannan, M. A., \& Jahan, N. (2014). Performance Analysis Of Frequency Reuse Schemes In Cellular Mobile Environment.

[4] Alabi, A. M., Taofiki, A. A., \& Olusegun, F. (2011). A combined scheme for controlling GSM network calls congestion. International Journal of Computer Applications, 14 (3).

[5] Deng, D. J., Lien, S. Y., Lee, J., \& Chen, K. C. (2016). On Quality-of-Service Provisioning in IEEE 802.11 ax WLANs. IEEE ACCESS, 4, 6086-6104.

[6] Rahman, M. (2015). QoS Provisioning Using Optimal Call Admission Control for Wireless Cellular Networks. arXiv preprint arXiv:1502.06329.
[7] Yan, Z., Liu, G., \& Su, R. (2015). Call admission control scheme with normalized quality of service metric in IEEE 802.16 networks. Wireless Communications and Mobile Computing, 15 (2), 309-321.

[8] Khdhir, R., Mnif, K., Belghith, A., \& Kamoun, L. (2016, November). An efficient call admission control scheme for LTE and LTE-A networks. InNetworks, Computers and Communications (ISNCC), 2016 International Symposium on (pp. 1-6). IEEE.

[9] Larasati, N., Kwee, W. K., Chong, S. C., \& Wee, Y. (2016). An analysis on quality of service enhancement in long term evolution networks: Past, present and future. Middle-East Journal of Scientific Research, 24 (3), 498-513.

[10] Ahmed, A. M. Y., Abdelghani, E. S. M., Babeker, R. F. A., \& Ali, Z. A. H. (2015). CALL ADMISSION CONTROL IN LTE (Doctoral dissertation, Sudan University of Science and Technology).

[11] El-Dolil, S. A., Al-Nahari, A. Y., Desouky, M. I. \& El-Samie, F. E. ( 2008). Uplink Power Based Admission Control in Multi-Cell Wcdma Networks With Heterogeneous Traffic: Progress in Electromagnetics Research B, 1, 115-134.

[12] Valko A. G. \& Campbell, A. T. (2000). "An efficiency limit of cellular mobile systems." Computer Communications Journal, vol. 23, no. 5-6, pp. 441-451.

[13] Singh, N., Sharma, R. L., \& Verma, P. (2016). Simulation And Performance Analysis Of Multi Hop Cellular Networks (Mncs) Over Single Hop Cellular Networks (Scns): A Queueing Model Approach. International Journal of Advances in Engineering \& Technology, 9 (3), 302.

[14] Siddiqui, A. F., Kumar, P., \& Tiwari, R. G. (2015). Reducing Handoff Blocking Probability in Wireless Cellular Networks-A Review.

[15] Singh, N., \& Bhardwaj, M. (2016). A Modified Channel Assignment Scheme for Multi-Hop Cellular Network: Minimum Delay. International Journal of multidisciplinary papers, 1 (1).

[16] Fang, Y. (2005). Performance Evaluation of Wireless Cellular Networks Under More Realistic Assumptions: Wireless Communications And Mobile Computing Wirel. Commun. Mob. Comput.; 5: 867-885 Published Online In Wiley Interscience (Www.Interscience.Wiley.Com). DOI: $10.1002 / \mathrm{Wcm} .352$. 